\title{
Asche im Wind
}

Wie man sich erzählend dem Leben entzieht

Brigitte Boothe

\section{Auf der Durchreise}

Blass sieht die alte Dame aus. Mit mattem Lächeln begrüßt mich Frau Anz ${ }^{1}$, fünfundachtzigjährig, im Alters- und Pflegeheim. Ihr Zimmer ist klein, schlicht und geradezu unpersönlich möbliert. Keine Erinnerungsstücke, kaum Fotos. Es wirkt, als hätte die Bewohnerin sich den Raum gar nicht zueigen gemacht, geschweige denn darin ausgebreitet. Ein Wartezimmer sozusagen. Die Frau mit dem kurz geschnittenen weißen Haar trägt eine Jacke und hat ihre Handtasche neben sich. Als sei sie auf der Durchreise, denke ich mir.

Sie hat sich bereit erklärt, mit mir über ihr Leben im Heim zu sprechen, und empfängt mich im Rollstuhl. Sie hat auf freiwilliger Basis mit unserer Zürcher Forschungsgruppe Kontakt aufgenommen; uns interessierte, wie es Bewohnern von Alters- und Pflegeheimen im Übergang vom privaten Zuhause ins Heim ergangen war, wie sie ihr Leben neu gestalteten, was es ihnen leicht oder schwer machte, sich neu einzurichten und ob dabei ein Bezug zu Religion oder Spiritualität eine Rolle spielte. ${ }^{2}$ Ich danke ihr für die Zeit, die sie mir schenkt, und frage, ob sie des Gesprächs wegen an diesem milden Frühlingsnachmittag auf eine Spazierfahrt im nahen Park verzichtet habe. "Nein.", sagt sie. "Ich war schon lange nicht mehr draußen. Mir wird so leicht kalt, und ich sehe nicht gut. Wenn die Sonne zu hell ist, schmerzen die Augen."Oder verpasst sie eine Veranstaltung im Heim? Auch das verneint sie: „Ich nehme selten an einem Anlass teil und bleibe lieber allein. "Und als ich etwas überrascht reagiere:

»Es ist nichts dabei, was mich anspricht. Ich kann nicht singen, Kartenspielen sagt mir nichts. Das Reden ist oft ermüdend für mich. Konzerte mit Volksliedern oder Unterhaltungsmusik sind für manche entspannend, aber ich werde unruhig. Es gibt gele-

1 Namen geändert.

2 Eva Baumann-Neuhaus/Brigitte Boothe/Ralph Kunz, Religion im Heimalltag. Ältere Menschen erzählen, Würzburg 2012. 
gentlich eine Art Erzählcafé, aber es fällt mir schwer zuzuhören.

Und ich selber ? - Was soll ich schon von mir selbst erzählen?«

Sie seufzt und ergänzt: „Und ich habe eben auch sonst noch Gründe. Ich sehe ziemlich schlecht, das Gehör hat nachgelassen. Und dann die Schwindelanfälle, die Blähungen; mir wird so leicht übel, und was weiß ich alles. Es soll vegetative Dystonie sein.«

Viele Personen, deren Beschwerden als "vegetative Dystonie» etikettiert werden, sind vorher persönlichen Belastungen ausgesetzt, mit denen sie sich im Vorfeld nur unzureichend hatten auseinandersetzen können. Die Diagnose verweist auf psychosomatische $\mathrm{Zu}$ sammenhänge. ${ }^{3}$ "Ganz schlimm ist das starke Schwitzen«, sagt Frau Anz und lächelt resigniert, »ich kann nicht unter die Leute gehen." Sie braucht den Rollstuhl, weil sie sich vor einigen Wochen einen dreifachen Beinbruch sowie einen Bänderriss zugezogen hat. Sie hat Schmerzen, geht aber sparsam mit lindernden Medikamenten um, weil sich anderenfalls die Verdauung verschlechtert.

\section{Ein Verlust von Heimat und Zugehörigkeit}

Ihre körperliche Verfassung ist zwar nicht lebensbedrohlich, doch fühlt sie sich matt und elend, von zahlreichen Missempfindungen und Unwohlsein gequält und belastet. "Am Anfang war es schwer für mich, hier zu sein, ich wollte nicht essen, all die fremden Leute waren mir ein Graus. Ich wolle niemanden kennenlernen." Es war eine große Überwindung gewesen, von Zuhause und vom Ehemann wegzugehen. "Aber es gab halt keine andere Lösung. " Denn sie litt damals täglich unter lang anhaltenden Schwindelanfällen, Übelkeit und Erbrechen. Es wurde immer schlimmer. Der Ehemann, selbst Diabetiker und dem Unterstützungsbedarf seiner Frau nicht gewachsen, hielt Ausschau nach einem Platz im Pflegeheim; schweren Herzens siedelte sie um. Ob sie enttäuscht war? Ob sie fürchtet, ihrem Mann nur noch eine Last zu sein, frage ich mich im Stillen.

Doch Frau Anz meint, es sei eine vernünftige Entscheidung gewesen. Der Mann - »er ist wirklich lieb« - komme auf Besuch. Aber

3 Die Diagnose einer vegetativen Dystonie ist ein pragmatisches Hilfsetikett. Sie ist medizinisch ungesichert; psychosomatische Zusammenhänge werden vermutet, das ist aber bisher nicht eindeutig geklärt. Die Diagnose findet üblicherweise Anwendung bei einer Reihe von Beschwerden, die als Fehlfunktionen des vegetativen Nervensystems verstanden werden. Es geht um Nerven, die nicht willentlich gesteuert werden und organische Symptome auslösen; das psychische Befinden ist oft vielfach und langwierig in Mitleidenschaft gezogen. 
sie ist nicht sicher, ob ihm die Begegnung dann Freude macht, denn sie klage über ihre Beschwerden und könne kaum damit aufhören. Eine Pflegerin habe schon zu ihr gesagt, sie solle nicht so viel klagen, ihr Mann würde das schlecht aushalten. Dass körperliches Leiden sie plagt, das bringt sie zur Sprache, denke ich; doch was ihr Kummer bereitet, findet keinen Ausdruck. Wie bei vielen organisch nicht vollständig erklärbaren Beschwerdebildern kann es auch hier der Körper sein, der Seelisches zur Darstellung bringt.

Wenn die beiden Töchter zu Besuch kommen, klage sie nicht. Sie seien ja auch mit dem eigenen Leben, mit ihren Berufen, Freunden, Hunden, Pferden, so beschäftigt, da wolle sie nicht eine zusätzliche Last sein. Ihr blasses Gesicht bleibt unbewegt. Sie sei sich selbst eine Last, äußert sie beiläufig. Ebenso beiläufig spricht sie davon, dass man stets neues Personal, oft fremdsprachig, um sich habe, da die Wechsel so häufig sind. Auf dem Gang trifft sie ständig demente Patienten, Kontakt sei unmöglich. Nun, das sei eben so. Die Betreuung sei durchaus in Ordnung, man gebe sich Mühe. Wie verbringt sie den Tag? Häufig mit dem Radio. Lesen kann sie nicht, Hörkassetten mag sie nicht. Musik macht sie nervös. Aber Wortsendungen schätzt sie, besonders wenn es um Biografisches geht, aber auch Diskussionen und Informationssendungen sind ihr wichtig.

\section{Die Kommunikation indifferenter Beiläufigkeit}

Es fällt mir schwer, das Gespräch in Gang zu halten. Andere Heimbewohner, die Kontakt mit uns aufgenommen hatten, gerieten rasch in ein engagiertes Erzählen, gestalteten lebhaft wichtige Begebenheiten, vergegenwärtigten mit emotionaler Beteiligung biografische Ereignisse und Situationen, durchlebten in ihren Schilderungen Glückliches und Trauriges noch einmal. Der Umstand, dass wir außerhalb des Kreises ihrer Angehörigen oder Freunde standen, erleichterte sogar oft das Erzählen und ermöglichte ihnen, legitimierte sie geradezu, sich auszubreiten und vor einem außenstehenden interessierten Hörer ihr Leben Revue passieren zu lassen und - manche sagten das - es als ein sinnvolles wertvolles Ganzes zu begreifen. Die erzählende Rede war charakteristischerweise bildhaft und bewegt. Frau Anz dagegen spricht mit indifferenter Beiläufigkeit, auch dann, wenn sie ihre Beschwerden erwähnt. Sie führt Themen nicht von sich aus weiter, sondern nimmt meine Fragen auf und gibt gleichsam Auskunft. Das Gespräch scheint nicht für sie selbst einen Wert zu haben; es ist eher so, dass sie sich bereit hält, mir für das wissenschaftliche Projekt Material zu liefern. Ich erfahre, dass Frau Anz aus städti- 
schem Milieu stammt, einen etwas älteren, noch lebenden Bruder hat, werde darüber informiert, dass die Familie seit mehreren Generationen ein erfolgreiches eigenes Geschäft betrieb, dass sie als Kind gern las und besonders tierlieb war. Ob Religion und Kirche in der Kindheit wichtig waren, will ich wissen und höre, dass die Zugehörigkeit zur reformierten Kirche Familientradition war, die aber äußerlich blieb, auch für sie selbst. Der Bruder machte akademische Karriere, Frau Anz absolvierte während des Zweiten Weltkriegs eine Hauswirtschaftslehre. Als junge Frau war sie in der Schweiz teils mit unterschiedlichen beruflichen Aufgaben unterwegs, unter anderem als nicht-examinierte Hilfskraft im ärztlichen Labor, dann in einer chirurgischen Praxis. Sie heiratete, das erste Kind kam auf die Welt; und als die zweite Tochter da war, beendete sie ihre berufliche Zeit.

\section{Abgeschlossene Lebenskapitel}

„Wozu bin ich auf der Welt? Woran kann ich glauben?«, das habe sie sich oft gefragt. Als die Töchter geboren wurden, war ihr der Sinn ihres Lebens nicht mehr zweifelhaft. Nun ist sie seit 60 Jahren verheiratet, und bis zum Eintritt ins Heim lebte sie in einem von ihrem Vater mitfinanzierten Haus; die Eltern hatten ihrerseits in der Nähe gewohnt; die Bindung an sie blieb lebenslang stark. Wie merkwürdig, dachte ich während des Gesprächs, vom Ehemann erzählt sie eigentlich gar nichts.

Frau Anz liebte Tiere und hatte Freude an diesen Hausgenossen. Wäre es nicht schön, im Heim ein Tier zu halten? Nein. Das kommt gar nicht in Frage: »Nein! Tiere! habe ich immer gehabt. Aber jetzt ist Schluss damit." Nicht etwa, weil sie für das Tier nicht würde sorgen können.Vielmehr geht es um die nachdrückliche und stolze Deklaration eines selbstbestimmten Abschieds vom einst geliebten Tierkontakt. Das Kapitel mit den Tieren ist in ihrem Lebensbuch jetzt einfach abgeschlossen. Das einst genossene Glück hat keinen Platz in der neuen Lebenssituation. Das gilt auch für anderes. Man könnte ja im Heim Geselligkeit genießen, manchmal feiern, sich mit Büchern abgeben, das Zimmer gemütlich machen; doch weigert sich Frau Anz, solche Ersatzbildungen und Neuorientierungen zu kultivieren: »Ach, eigentlich kann man ja nicht mehr richtig in Kontakt kommen. Ich habe es auch mal probiert. Da gab es eine ganz sympathische Person, aber auf die Länge sagt es mir einfach nichts mehr.«Anderen biografischen Erzählern bedeutete die emotionale Vergegenwärtigung vergangenen Lebens viel: »Noch jetzt sehe ich 
vor mir ...", "Ich weiß noch wie heute ...«.»Diese vorwurfsvolle Stimme vergesse ich nicht ...", »Der Duft der Wiesen im Sommer ...", "Einmal hat mein Vater mich an Fasnacht zum Umzug mitgenommen. Wir waren als Clowns verkleidet, ich war so stolz ..... Die Erzähler machen gewöhnlich kommunikativ deutlich, in welcher Weise und wie intensiv die wiedererlebten Ereignisse Macht und Einfluss auf ihr Gemüt ausüben. Frau Anz kommuniziert das Gegenteil. Die Beiläufigkeit der oft nur summarisch und lakonisch skizzierten biografischen Elemente macht sie zu abgelegten Versatzstücken einer entfärbten Vergangenheit. Abgeschoben, weggeschoben, abgestorben. »Ich habe von Zuhause wenig mitgenommen«, sagt sie, auf ihr karges Zimmer blickend. Es ist ja vorbei. Wer unter Schwindel leidet, findet keinen sicheren Halt, wer schlecht sieht, hat keine Orientierung, wem immer wieder übel ist und das Essen schlecht verdaut, dem bekommt die Lebensnahrung nicht. Das Leben ist unbekömmlich geworden.

\section{Eine Lichtgestalt}

Nicht ganz. Da sind noch monatliche Beratungsgespräche mit einer erfahrenen, vertrauenswürdigen, liebenswürdigen und hochgeschätzten Psychologin. "Ja, die Frau Lienert ist eine kluge Person, und sie will mir helfen. Sie hilft bestimmt vielen." Frau Anz äussert sich positiv über die Beraterin und entzieht sich zugleich ihrem Einfluss. Und dann geht es noch um eine Licht- und Heilsgestalt: Sie hatte Kontakt zu einem Pfleger, der sie beeindruckte. Er war für sie die Verkörperung der Menschenliebe, aber auch ein Leidensmann, der, selbst schwer erkrankt, dennoch alle mit seiner Liebesfähigkeit beschenkte:

„Er war wie ein Wunder. Sein Lächeln. Sein Blick. Er hatte dann aber eine aggressive Leukämie gehabt und ein ganzes Jahr damit gekämpft. Er hat mir gefehlt in dieser Zeit. Und wir im Heim haben Angst gehabt, dass er die Krankheit nicht überlebt. Jetzt ist er aber zweimal wieder gekommen. Das waren solche Lichtblicke.«

Ein Wunder ist, dass dieser Mann die tödliche Krankheitsattacke überlebte. Der Segen, das Heilende, das von ihm ausging, hielten ihn selbst am Leben. Sie stellt ihn dar als jemanden, der todgeweiht war und den Tod vorläufig besiegte: »Ja, der hat eine Ausstrahlung, eine solche Ausstrahlung von Liebe. Mit allen ist er lieb.« Doch auch 
wenn er Frau Anz gefehlt hat und sie über ihn mit etwas stärkerer emotionaler Beteiligung spricht, formuliert sie summarisch und eher aus der Distanz einer Zeugin. Die fortgesetzte Verwendung der Worte "lieb « und "Liebe« zielt zwar auf Bekräftigung und Steigerung, hat aber eher einen pauschalisierenden Effekt mit dem Anklang des Beliebigen. Im übrigen ist Frau Anz Skeptikerin und Agnostikerin, äußert aber eine gewisse wohlwollende Toleranz für sympathische und umgängliche Repräsentanten der Kirche; es fällt ihr sogar ein humor- und gemütvoller Pfarrer aus der Kindheit ein und ein anderer, der bei der Beerdigung der Eltern amtierte. Religion und Gottesglaube seien aber kein Thema für sie. "Ein liebender Gott ist eine lächerliche Illusion«, meint sie und wird bei diesem Gedanken spürbar unwillig.

\section{"Ich möchte sterben $«$}

Schließlich kommt es zu einem Bekenntnis: "Ja, wissen Sie, also jetzt sage ich es Ihnen, ja, ich möchte sterben. Ich habe schon Kontakt mit der Sterbehilfe aufgenommen." Der Tod sei nichts, nach dem Tod komme nichts. Nur eine anonyme Gemeinschaftsstätte auf dem Friedhof komme für sie in Frage, dort sei ihre Asche zu verstreuen. "Ich finde mich halt gar nicht wichtig. Auch mein Mann will dann so eine anonyme Stätte. Natürlich soll auch kein Pfarrer kommen, am besten gar keine Abdankung." Das Ende ihres Lebens möge selbstbestimmt sein. Das Leben hatte einst Sinn. Doch das ist jetzt vorbei. Was gut war, ist verloren. Es soll und darf dafür keinen Ersatz geben. Das Leben im Heim ist keine Phase, die neu und initiativ gestaltet würde. Die Gegenwart wird verworfen. Zukunft ist eine Illusion. Eine religiöse Heimat kann es nicht geben. Es bleibt die Souveränität der Selbstpreisgabe und der Selbstauslöschung. Ein Lebensabschluss wie Asche im Wind.

Und doch stellt sich der Lebens- und Selbstentwertung der Frau Anz die Lichtgestalt eines Pflegers entgegen, der - wie Jesus im Neuen Testament - den Tod besiegt hat. Im Zeichen dieser Lichtgestalt: Könnte hier nicht doch etwas Neues entstehen? Etwas anderes als die Absicht, eine Sterbehilfeorganisation in Anspruch zu nehmen? Zwar hat sie sich im Heim in die Isolation begeben, zwar hat sie sozusagen mit allem abgeschlossen, zwar entwickelt sie in Vorstellung und Fantasie die völlige Auslöschung, aber sie tut das ja immerhin im Gespräch. Sie hat ja immerhin aktiv mit unserer Forschungsgruppe Kontakt aufgenommen. 
Der Wunsch nach beschleunigtem Sterben mag den Wunsch verdecken, angenommen zu sein und und in neuer Integrität, gleichsam erlöst und geheilt, neu zu beginnen. ${ }^{4}$

Der Ruf "Hilf mir beim Sterben" verbirgt, folgt man de M'Uzan, häufig den Wunsch: »Schenk mir neues Leben. "Frau Anz vermittelt eine zweifache Botschaft: Einerseits will sie, dass der Suizidwunsch akzeptiert wird. Andererseits wünscht sie Begleitung in einer Lebenssituation, die sie als nicht lebenswert erachtet. Sie gibt zu verstehen, dass ihr Leben abgeschlossen sei. Doch zieht sie ihr Gegenüber ins Gespräch über Lebensmöglichkeiten, Wert des Lebens und Lebensende.

\section{Schenk mir neues Leben}

Es kann die Hoffnung auf neues Leben sein, die sich im Sterbewunsch verbirgt. Die Entwertung und Entfärbung der biografischen Vergangenheit und der jetzigen Möglichkeiten kann eine mächtige Abwehrbewegung sein gegen den Wunsch nach Lebensfülle und die Trauer um eine verlorene Heimat. Franz Kafkas erste Zeilen seiner Parabel Heimkehr (1920) - eine düstere Verwandlung der neutestamentlichen Parabel vom verlorenen Sohn - gestalten sprachlich eine radikale Trostlosigkeit, die der Hoffnung auf liebevolle und festliche Aufnahme beim Vater Hohn spricht:

»Ich bin zurückgekehrt, ich habe den Flur durchschritten und blicke mich um. Es ist meines Vaters alter Hof. Die Pfütze in der Mitte. Altes, unbrauchbares Gerät, ineinander verfahren, verstellt den Weg zur Bodentreppe. Die Katze lauert auf dem Geländer. Ein zerrissenes Tuch, einmal im Spiel um eine Stange gewunden, hebt sich im Wind. $\ll^{5}$

Von abgegriffenen Altertümern ist auch in Jean Pauls Erzählung die Rede, in der es am Ende um das Sterben des "Schulmeisterlein Wutz« geht:

"Ich will mein Versprechen der Spezifikation halten: auf dem Deckbette lag eine grüntaftne Kinderhaube, wovon das eine Band abgerissen war, eine mit abgegriffnen Goldflitterchen überpichte Kinderpeitsche, ein Fingerring von Zinn, eine

4 Vgl. Michel de M'Uzan, De l'art à la mort. Itinéraires psychanalytiques, Paris 1983.

5 Franz Kafka, Heimkehr, in: ders., Kurze Prosa, Erzählungen. 16 Interpretationen, hg. v. Ralf Sudau, Stuttgart 2007, 68-69, hier 68. 
Schachtel mit Zwerg-Büchelchen in 128-Format, eine Wanduhr, ein beschmutztes Schreibbuch und ein Finkenkloben fingerlang. Es waren die Rudera und Spätlinge seiner verspielten Kindheit. Die Kunstkammer dieser seiner griechischen Altertümer war von jeher unter der Treppe gewesen - denn in einem Haus, das der Blumenkübel und Treibkasten eines einzigen Stammbaums ist, bleiben die Sachen jahrfunfzig lang in ihrer Stelle ungerückt-; und da es von seiner Kindheit an ein Reichsgrundgesetz bei ihm war, alle seine Spielwaren in geschichtlicher Ordnung aufzuheben, und da kein Mensch das ganze Jahr unter die Treppe guckte als er: so konnt' er noch am Rüsttage vor seinem Todestage diese Urnenkrüge eines schon gestorbenen Lebens um sich stellen und sich zurückfreuen, da er sich nicht mehr vorauszufreuen vermochte. $[\ldots][U]$ nd die Strahlen der auferstehenden Kindheit spielten, wie des gemalten Jesuskindes seine im Stall, an den düsteren Winkeln! « ${ }^{6}$

Hier ist es die Vergnügen schaffende Erinnerungskunst, sozusagen an den Konkreta der noch lebenden und bestrahlten Gegenstände sich bereichernd. Die Sterbeszene betont eindringlich die retrospektive Verklärungskunst, die dem biografischen Erinnern höchst erfreulich eignen kann. Die Gegenstände sind abgenutzt, doch das ist bei Jean Paul kein Grund, sie zu entwerten. Im Gegenteil. Das »Zurückfreuen« schafft Bindung und Verbindung. Kafkas Blick auf die abgelebten "Altertümer» hingegen schafft Trennung. Eine Trennung, die auch in der Sprache von Frau Anz wirksam wird. Sie spricht, wie Kafka, von Abgestorbenem, von Dingen, die kein neues Leben mehr gewinnen können. Doch wie in Kafkas Parabel ist die Sehnsucht nach neuem Leben und nach liebevoller Wertschätzung spürbar. Die Sehnsucht ist spürbar, doch hielt Frau Anz an ihrem Plan fest. Sie nahm Sterbehilfe in Anspruch.

Bekanntlich können Personen, die sozusagen vorauseilend die eigene Biografie im Duktus des Abgestorbenen artikulieren und eine Abwehrbewegung des Entwertens und der Selbstentwertung vornehmen, hineingelockt werden ins Erzählen, indem man die Rollen tauscht und indem die beratende, begleitende oder seelsorglich unterstützende Person selbst Erzählungen anbietet. Erzählungen, die Spannung erzeugen und Lust auf Mehr machen, Erzählungen unterschiedlicher Provenienz, Gattung und Stilrichtung, wie sie kulturell tradiert sind oder wie sie zur psychischen Verfassung der

\footnotetext{
${ }^{6}$ Jean Paul, Leben des vergnügten Schulmeisterlein Maria Wutz im Auenthal. Eine Art Idylle. Anhang zu: Die unsichtbare Loge, Frankfurt a.M. 1995, 54-55.
} 
Person passen. Scheherazade ist das gelungen, 1001 Nächte lang. Der König konnte auf die nächtlichen Unterhaltungen nicht verzichten. Und während er Spaß hatte, kam es bei diesem Protagonisten zu einem persönlichen Veränderungs- und Bildungsprozess.

Eine Wunschvorstellung: Während Frau Anz den Geschichten zuhört und mit der Erzählerin darüber spricht, gewinnt sie eine Sprache für noch sprachlose Aspekte ihres Selbst- und Weltverhältnisses.

Frau Anz wollte sich verflüchtigen, doch verschwand sie nicht einfach, auch nicht aus meiner Erinnerung. Mit der "Reisebekanntschaft «, die ich machen durfte, wollte ich im Gespräch bleiben, auch wenn sie nicht antwortet.

- Brigitte Boothe war bis 2013 ordentliche Professorin für Klinische Psychologie, Psychotherapie und Psychoanalyse an der Universität Zürich. Seit ihrer Emeritierung wirkt sie nebst ihrer Forschungstätigkeit in der Gemeinschaftspraxis Bellevue, Zürich. 